\title{
SUPERCRITICAL FLUID INJECTION OF NONVOLATILES WITH RESONANT TWO PHOTON IONIZATION DETECTION IN SUPERSONIC BEAM MASS SPECTROMETRY
}

\author{
D. M. Lubman, C. H. Sin, H. M. Pang \\ Department of Chemistry, University of Michigan \\ Ann Arbor, MI 48109
}

\begin{abstract}
Supercritical fluids of $\mathrm{N}_{2} \mathrm{O}$ and $\mathrm{CO}_{2}$ are used to solubilize nonvolatile compounds for supersonic jet expansions. R2PI is then used to ionize these compounds in a time of flight mass spectrometer. By tuning the dye laser wavelength a cold wavelength ionization spectrum can be obtained that is characteristic of that species.
\end{abstract}

\section{INTRODUCTION}

In our work resonant two-photon ionization (R2PI) in supersonic jets is used as a means of optically selecting ions for mass spectrometry. The use of supersonic beam injection provides internally ultracold molecules with sharp spectral features for chemical analysis for molecules whose spectra would otherwise exhibit broad, unresolvable contours at room temperature. Our present work focuses on the use of this method for analysis of small biological species based upon the combination of wavelength selectivity and the high efficiency for producing molecular ions by the R2PI technique. One method investigated for use in solubilizing nonvolatile and thermally labile molecules involves expansions of supercritical fluids of $\mathrm{CO}_{2}$ and $\mathrm{N}_{2} \mathrm{O}$ at up to 400 atm backpressure into a mass spectrometer at $10^{-5}$ torr. Direct liquid introduction (DLI) techniques developed for HPLC/MS could also be used for introduction of nonvolatiles into vacuum. However, the much higher volatility of most gases that might be used in SFI $\left(\mathrm{CO}_{2}, \mathrm{~N}_{2} \mathrm{O}, \mathrm{NH}_{3}\right.$, etc.) provides a significant advantage over HPLC or liquid introduction into a mass spectrometer since the supercritical fluid immediately converts into a beam of molecules after introduction into vacuum, whereas DLI may require significant heating of the nozzle in order to prevent large clusters or crystals of material from condensing out upon expansion. In addition, SFI can utilize carrier gases which have a low enough number of internal modes to form a molecular beam so that significant cooling of the seeded material can be achieved. The expansion of this ultradense jet can thus provide sharp spectral features for analysis of nonvolatiles in the reservoir at room temperature which would normally require significant heating for volatilization into the gas phase.

\section{EXPER IMENTAL}

The experimental set-up is described elsewhere (1). It consists of a differentially pumped vacuum system with a ToF mass 
spectrometer sitting vertically on top of the chamber. The ultraviolet laser beam enters the chamber through quartz windows and R2PI is produced in the acceleration region of the TOFMS. The novel feature of this experiment is that a 150-200 um orifice can be used to obtain high on-axis density in the jet even with $400 \mathrm{~atm}$ backpressure using a specially designed high pressure pulsed injection valve. Using this valve in conjunction with efficient $\mathrm{LN}_{2}$ cryopumping, a pressure of $\sim 10^{-5}$ torr can be maintained in the mass spectrometer. The laser source is a Quanta-Ray Nd:YAG pumped laser which is frequency doubled in $K \mathrm{~K}^{*} \mathrm{P}$ to produce tunable near-UV light. The supercritical fluid pressure of $\mathrm{CO}_{2}$ and $\mathrm{N}_{2} \mathrm{O}$ is provided by a Varian 8500 syringe pump.

\section{RESULTS AND DISCUSSION}

In Figure 1a is shown a spectrum of the $S_{0} \rightarrow S_{1}$ transition of carbazole expanded from supercritical $\mathrm{CO}_{2}$ at $198 \mathrm{~atm}$ back pressure and $40^{\circ} \mathrm{C}$. The melting point of carbazole is $245^{\circ} \mathrm{C}$ and at $40^{\circ} \mathrm{C}$ essentially no signal is observed from a 1 atm back pressure of $\mathrm{Ar}$. In Figure $1 \mathrm{~b}$ is a spectrum of carbazole obtained using 1 atm of $\mathrm{CO}_{2}$ at $180^{\circ} \mathrm{C}$. This spectrum was taken by use of a special pulsed solenold valve capable of heating to at least $300^{\circ} \mathrm{C}$. However, using supercritical fluid expansions of $\mathrm{CO}_{2}$, a low temperature can be used to seed carbazole into a molecular beam, though it has a fairly high melting point.

Both spectra in Figure 1 have absorptions that are broader than are observed with Ar since the rotational population is not as effectively cooled by $\mathrm{CO}_{2}$ as by $\mathrm{Ar}$. This result is expected since the terminal Mach number ${ }^{2}\left(M_{T}\right)$ depends on the heat capacity ratio, $r=C_{p} / C_{V}$, which is larger for $\mathrm{Ar}$ than for $\mathrm{CO}_{2}$. In essence, the monatomic gas has no vibrational and rotational degrees of freedom in which to store energy and thus reaches its final translational temperature more quickly than in a polyatomic gas. This incomplete cooling of the beam leaves a significant number of molecules in excited rovibronic states, so that resonant ionization of these molecules makes a substantial contribution to the signal seen as the laser is swept over a relatively broad spectral range which is why the background baseline never goes to zero.

The mass spectra obtained for the laser photoionization of various PNAH's in a time-of-flight mass spec. are shown in Figure 2, which is a mass spectrum of acenaphthene, carbazole, phenanthrene, pyrene, and tetracene obtained from a supercritical expansion of 350 atm $\mathrm{CO}_{2}$ at $\mathrm{T}=40^{\circ} \mathrm{C}$ using $266 \mathrm{~nm}$ as the ionization source. These compounds all dissolve strongly in the supercritical fluid even at $40^{\circ} \mathrm{C}$ even though tetracene has a melting point of $300^{\circ} \mathrm{C}$. We have also explored a number of small biologicals including tryptophan, tyramine, phenoxazine and benzimidazole which can be solubilized by supercritical $\mathrm{CO}_{2}$ or $\mathrm{N}_{2} \mathrm{O}$ at essentially room temperature and have obtained wavelength dependent spectra for benzimidazole. The unique features of the mass spectra obtained are 1) an operating temperature only slightly higher than room temperature can be used to dissolve the nonvolatile or thermally labile compounds and 2) 
with the use of laser ionization, molecular ions are produced without fragmentation.

Our most recent work utilizes derivatization as a means of increasing the solubility of polar biological species in supercritical $\mathrm{CO}_{2}$ or $\mathrm{N}_{2} \mathrm{O}$ by decreasing the polarity of the - $\mathrm{COOH}$ group. We have been able to solubilize various catecholeamines and the ir metabolites by converting the $-\mathrm{COOH}$ to a $-\mathrm{COOCH}_{3}$ group. These derivatized compounds readily dissolve in the fluid and can be ionized at $280 \mathrm{~nm}$. This is expected since the derivatization does not strongly change the absorption or ionization properties of the original molecule. We have recently obtained a wavelength ionization spectrum of the methyl ester derivative of indole-3acetic acid using supercritical $\mathrm{CO}_{2}$ expansion and its spectrum compares favorably with that of the underivatized compound.

\section{REFERENCES}

1. C. H. Sin, H. M. Pang, D. M. Lubman, and J. Zorn, Anal. Chem., $58,487(1986)$.

2. H. M. Pang, C. H. Sin, D. M. Lubman, and J. Zorn, Ana 1. Chem., $58,1581(1986)$.
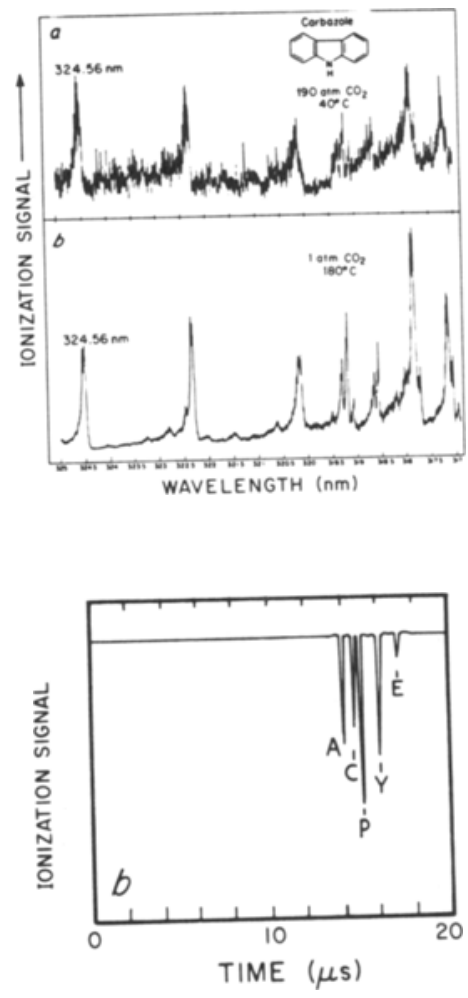

Fig.1 R2PI spectrum of carbazole expanded in a supersonic jet of (a) supercritical $\mathrm{CO}_{2}$ at $190 \mathrm{~atm}$ and $40^{\circ} \mathrm{C}$ and (b) 1 atm $\mathrm{CO}_{2}^{2}$ at $180^{\circ} \mathrm{C}$.
Fig.2 Time-of-flight mass spectra of acenaph thene (A), carbazole(C), phenanthrene $(P)$, pyrene $(Y)$ and tetracene(E) expanded from a jet at $350 \mathrm{~atm} \mathrm{CO}_{2}$ and $40^{\circ} \mathrm{C}$. 